\title{
Hepatitis E Virus Infection in Dromedaries, North and East Africa, United Arab Emirates, and Pakistan, 1983-2015
}

\author{
Andrea Rasche, Muhammad Saqib, \\ Anne M. Liljander, Set Bornstein, Ali Zohaib, \\ Stefanie Renneker, Katja Steinhagen, \\ Renate Wernery, Mario Younan, Ilona Gluecks, \\ Mosaad Hilali, Bakri E. Musa, Joerg Jores, \\ Ulrich Wernery, Jan Felix Drexler, \\ Christian Drosten, Victor Max Corman
}

A new hepatitis E virus (HEV-7) was recently found in dromedaries and 1 human from the United Arab Emirates. We screened 2,438 dromedary samples from Pakistan, the United Arab Emirates, and 4 African countries. HEV-7 is long established, diversified and geographically widespread. Dromedaries may constitute a neglected source of zoonotic HEV infections.

$\mathrm{H}$ epatitis E virus (HEV) is a major cause of acute hepatitis worldwide (1). Four HEV genotypes belonging to the species Orthohepevirus $A$ are commonly found in humans (HEV-1 through HEV-4). Genotypes 1 and 2 seem to be restricted to humans, whereas genotypes 3 and 4 also occur in domesticated and wild animals. Zoonotic transmission by ingestion of contaminated meat, mainly from pigs, is the most likely zoonotic source of infection (1).

Recently, HEV sequences were reported from 3 dromedaries sampled in the United Arab Emirates (UAE) in 2013 and were classified as a new orthohepevirus A genotype, HEV-7 (2,3). Afterwards a human patient also from the UAE who had chronic hepatitis after liver transplantation was shown to carry HEV-7 (3,4). Until now,

Author affiliations: University of Bonn Medical Centre, Bonn, Germany (A. Rasche, J.F. Drexler, C. Drosten, V.M. Corman); University of Agriculture, Faisalabad, Pakistan (M. Saqib, A. Zohaib); International Livestock Research Institute, Nairobi, Kenya (A.M. Liljander, J. Jores); National Veterinary Institute, Uppsala, Sweden (S. Bornstein); EUROIMMUN AG, Lübeck, Germany (S. Renneker, K. Steinhagen); Central Veterinary Research Laboratory, Dubai, United Arab Emirates (R. Wernery, U. Wernery); Vétérinaires Sans Frontières Germany, Nairobi (M. Younan, I. Gluecks); Cairo University, Giza, Egypt (M. Hilali); Ministry of Science and Communication, Khartoum, Sudan (B.E. Musa); University of Bern, Bern, Switzerland (J. Jores); German Centre for Infection Research, Bonn (J.F. Drexler, C. Drosten, V.M. Corman)

DOI: http://dx.doi.org/10.3201/eid2207.160168 knowledge on HEV-7 and its zoonotic potential relied on these 2 studies, which provide no insight into the prevalence and distribution of HEV-7. To determine the geographic distribution of HEV-7, we conducted a geographically comprehensive study of HEV-7 prevalence in dromedaries by testing 2,438 specimens sampled in 6 countries during the past 3 decades.

\section{The Study}

Serum and fecal samples were collected from dromedary camels in the UAE, Somalia, Sudan, Egypt, Kenya, and Pakistan during 1983-2015 (5-7). A total of 2,171 serum samples and 267 fecal samples were tested for HEV RNA by using reverse transcription PCR (RT-PCR) as previously described (8). Seventeen samples were positive for HEV RNA: $12(0.6 \%)$ of 2,171 serum samples and $5(1.9 \%)$ of $267 \mathrm{fe}-$ cal samples (Table). Positive samples originated from UAE, Somalia, Kenya, and Pakistan and dated to 1983 (Figures 1, 2). Viral loads were measured by using real-time RT-PCR (9) calibrated on the basis of the World Health Organization International Standard for HEV RNA (10). Viral RNA concentrations ranged from $3.2 \times 10^{4}$ to $3.6 \times 10^{7} \mathrm{IU} / \mathrm{g}$ in feces and $6.2 \times 10^{2}$ to $8.3 \times 10^{6} \mathrm{IU} / \mathrm{mL}$ in serum.

We sequenced a 283 -nt fragment of the RNA-dependent RNA polymerase gene of all positive samples for phylogenetic analyses. All camel HEV clustered in a monophyletic clade with the human HEV-7 sequence (Figure 2), supporting the classification of camel-associated HEV to a separate Orthohepevirus A genotype (11).

African viruses from Somalia and Kenya formed a monophyletic clade, whereas viruses from UAE and Pakistan were intermixed (Figure 2). Distances based on nucleotide identities were calculated for all sequences from this study and 1 reference strain from each orthohepevirus A genotype as defined by Smith et al. (11). This subset of references comprised GenBank accession nos. M73218 (HEV-1), M74506(HEV-2), AF082843 (HEV-3), AJ272108 (HEV-4), AB573435 (HEV-5), AB602441 (HEV-6), and KJ496143 (HEV-7). Nucleotide diversity was remarkable among viral sequences from dromedaries, reaching a maximum distance of $22.7 \%$, compared with a maximum distance of $29.9 \%$ among all genotypes. The internal distance among the African viruses was $14.2 \%$, compared with $17.4 \%$ distance within viruses from UAE and Pakistan. The African viruses were $16.7 \%-22.7 \%$ distant from UAE and Pakistan viruses, which corresponds to the distance threshold of $22 \%-25 \%$ 
Table. Sample characteristics and detection rates of orthohepevirus Agenotype 7 in 6 countries, 1983-2015

\begin{tabular}{|c|c|c|c|c|}
\hline \multirow[b]{3}{*}{ Country } & \multirow[b]{3}{*}{ Time period } & \multicolumn{3}{|c|}{ No. positive/no. tested (\% positive) } \\
\hline & & \multicolumn{2}{|c|}{ Virus RNA } & \multirow[b]{2}{*}{ Antibodies } \\
\hline & & Serum & Feces & \\
\hline Sudan & 1983 & $0 / 60$ & & $15 / 35(42.9)$ \\
\hline Somalia & 1983-1984 & $1 / 105(0.9)$ & & $14 / 35(40.0)$ \\
\hline Egypt & 1997 & $0 / 50$ & & $22 / 35$ (62.9) \\
\hline Kenya & 1992-2015 & $2 / 889(0.2)$ & & $11 / 35(31.4)$ \\
\hline United Arab Emirates & 2013 & $1 / 500(0.2)$ & $5 / 267(1.9)$ & $13 / 35(37.1)$ \\
\hline Pakistan & 2012-2015 & $8 / 567(1.4)$ & & $21 / 35(60.0)$ \\
\hline Total & & $12 / 2,171(0.5)$ & $5 / 267(1.9)$ & $96 / 210(45.7)$ \\
\hline
\end{tabular}

that separates the prototype HEV-4 sequence from HEV-5 and HEV-6 prototype sequences. This finding suggests that HEV-7 is a strongly diversified clade of viruses that might need to be further subclassified.

HEV-7 was recently shown to belong to the same serotype as HEV-1-4 (12). Therefore, we conducted a preliminary serologic analysis with a subset of 210 specimens (35 per country) by adapting a human HEV ELISA (EUROIMMUN, Lübeck, Germany) for application with camel serum. Serum was tested at a 1:100 dilution. The signalto-noise ratio was optimized by normalizing the optical density (OD) of test samples against ODs of a reference serum included in every run (online Technical Appendix Figure, http://wwwnc.cdc.gov/EID/article/22/7/16-0168Techapp1.pdf).

For confirmation of ELISA results and to determine an appropriate ELISA cutoff, we tested 56 samples covering the complete range of OD ratios by adapting the recomLine Immunoblot (MIKROGEN, Neuried, Germany). Thirtytwo samples reacted against $\geq 2$ of the presented antigens and were therefore ranked positive in the Immunoblot. All tested samples with ELISA OD ratios $>0.46$ were positive by immunoblot, whereas only 7 of 31 tested samples below this value were positive by immunoblot (online Technical Appendix Figure). Subsequently we set an ELISA cutoff of 0.46 . Using this cutoff, we found $96(46 \%)$ of the 210 serum samples originating from all 6 countries were positive (Table), which is comparable with the seroprevalences typically observed in pigs that are known zoonotic reservoirs for HEV-3 in developed countries (13). The percentage of ELISA-positive serum samples ranged from $31 \%$ in Kenya to $63 \%$ in Egypt but did not differ significantly among all 6 countries $\left(\mathrm{p}=0.1\right.$, Yates' $\chi^{2}$ test). These results suggest a wide occurrence and high prevalence of $\mathrm{HEV}$ in dromedaries.

\section{Conclusions}

We investigated HEV-7 infection in dromedaries. The broad spatial extent, the high diversity of HEV-7 in dromedaries, and the detection of HEV-RNA in a sample collected in 1983 suggest a long evolutionary history of HEV7 in dromedaries.

Our study has some limitations. First, although most tested dromedaries seemed healthy, no detailed health

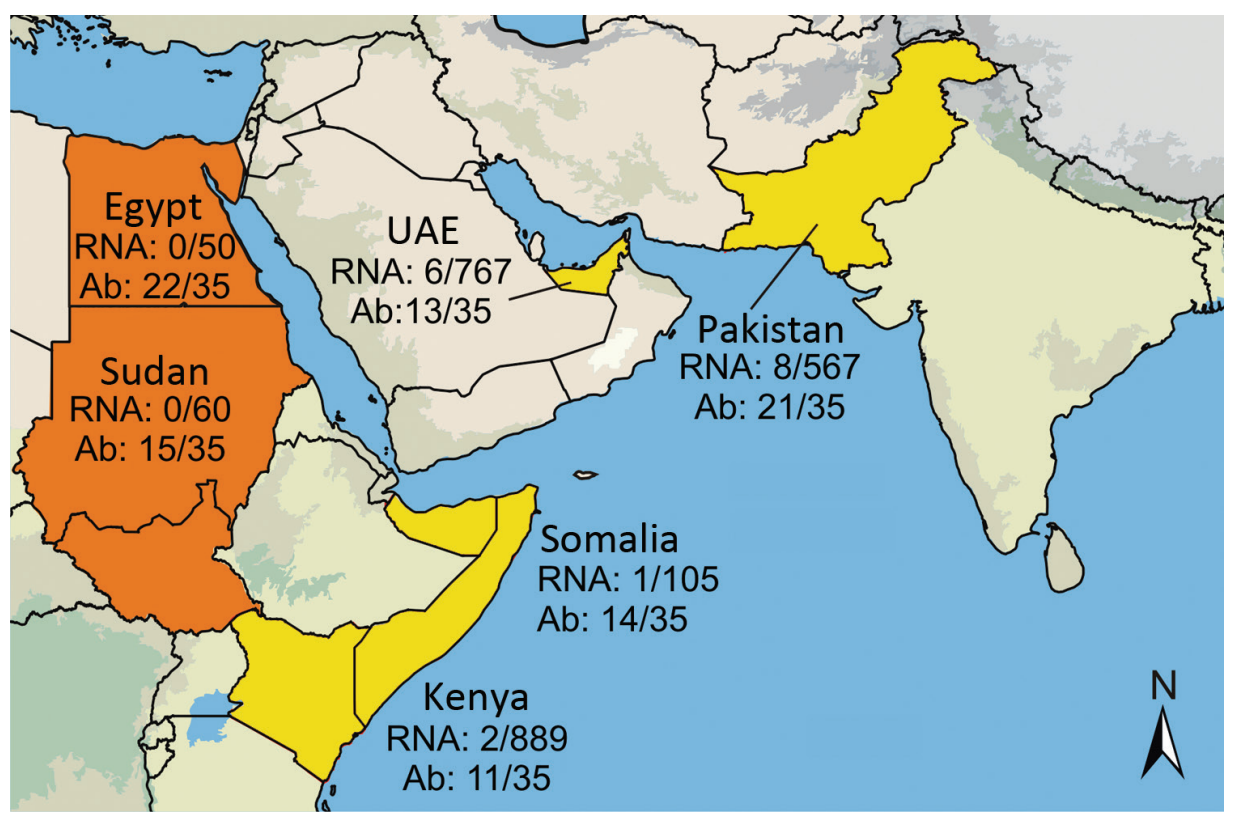

Figure 1. Six countries studied for hepatitis $E$ virus (HEV) infection in dromedary camels, 1983-2015. Number of tested and number of HEV-7 RNA-positive samples or Ab-positive samples are given next to the study sites: Egypt, Sudan (today separated into Sudan and South Sudan), Kenya, Somalia, UAE, and Pakistan. Countries with both HEV-7 RNA and Ab detection are in yellow; countries with only $\mathrm{Ab}$ detection are in orange. $\mathrm{Ab}$, antibody; UAE, United Arab Emirates; Map was created by using Quantum GIS (http://qgis.osgeo.org) and data from http://www.natural earthdata.com. 
HEV Infection in Dromedaries

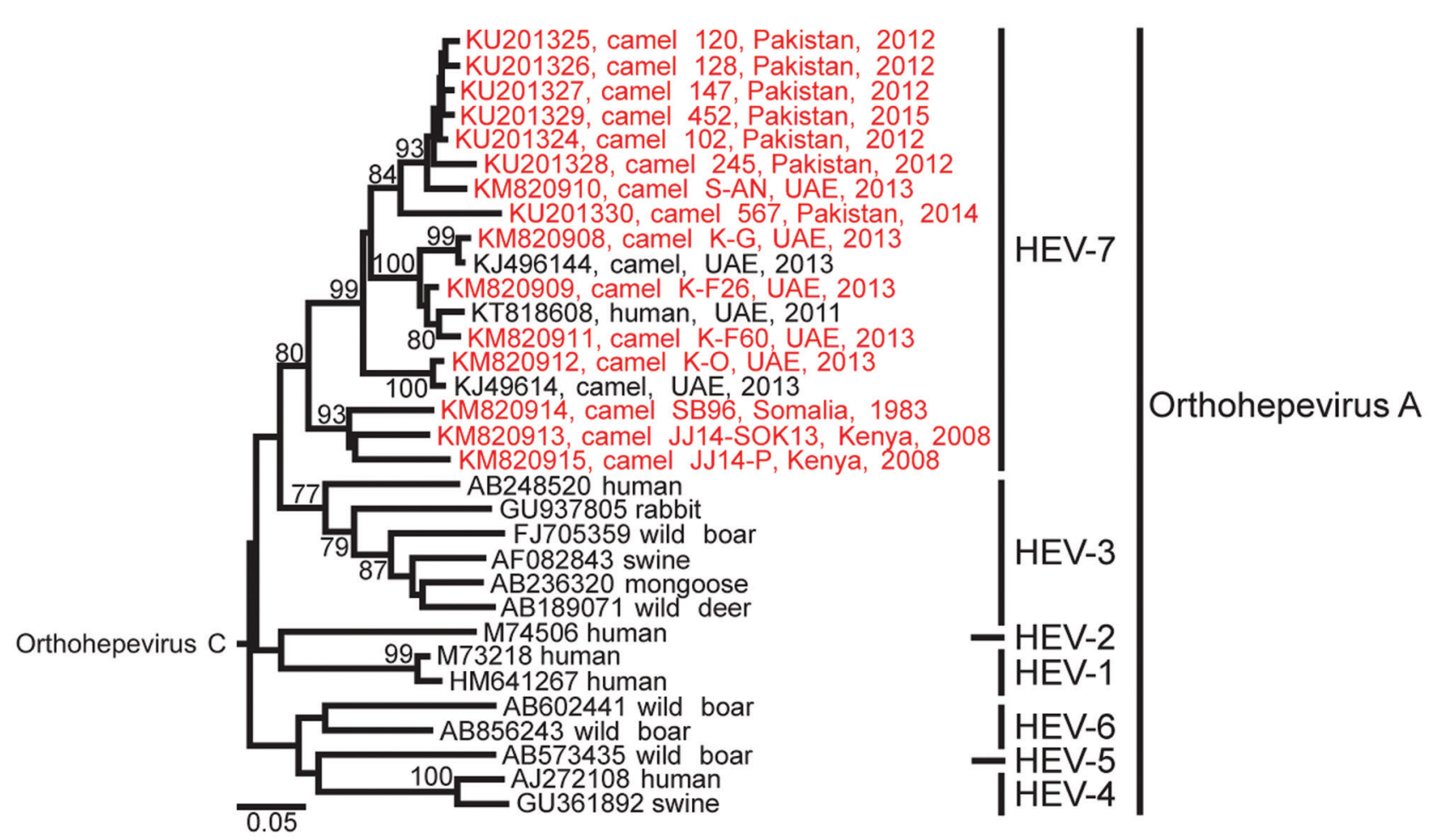

Figure 2. Phylogenetic analysis of Orthohepevirus $A$ sequences. The analysis comprised partial hepatitis $E$ virus (HEV) sequences (283 nt from the RNA-dependent RNA polymerase region) from this study, representatives of Orthohepevirus A genotypes 1-7 and Orthohepevirus C (GenBank accession no. GU345042) as an outgroup. The phylogenetic tree was calculated with MEGA 6.0 (http://www.megasoftware.net) by using the neighbor-joining algorithm and a nucleotide percentage distance substitution model. Bootstrap values $(\%)$ of 1,000 repetitive analyses $>75$ are shown next to the nodes. New camel HEV sequences obtained in this study are in red. Scale bar represents the genetic distance. All sequences obtained in this study are deposited in GenBank (accession nos. KM820907-KM820915 and KU201324-KU201330). UAE, United Arab Emirates.

information from the RNA-positive animals was available. Second, we studied limited genome fragments that prevented formal classification into genome subtypes (14). Third, although we used 2 different antibody detection methods, the antibody prevalence in camels should be confirmed by larger studies including virus neutralization studies to determine potential genotype variability.

Investigations of camelids other than dromedaries could help to further elucidate the geographic and evolutionary origin of HEV-7. Furthermore, other wild or domestic ungulates with close contact to dromedaries could be investigated to assess the host range of HEV-7. Human infection with HEV is common in all studied areas (1). On the basis of clinical observations and HEV antibody detection tools, several HEV outbreaks mainly linked to water contamination or poor hygienic circumstances have been described for Pakistan, Sudan, Somalia, and Egypt. For Kenya and UAE, data about HEV prevalence is scarce (1). In large parts of the Middle East, human infections are unlikely to be caused by contact with swine or consumption of pork for cultural reasons. Even in Saudi Arabia, where pork is absent in diet, blood donors have antibodies at proportions of up to $18.7 \%$ (1). Thus, most HEV infections in the Middle East are assumed to be caused by nonzoonotic genotypes 1 and 2. However, our study and previous studies (12) showed that HEV-7 and other human genotypes form 1 serotype, suggesting a lack of discrimination in seroprevalence studies.

The human HEV seroprevalence in the Middle East region might in fact be caused by HEV-7 infection. Furthermore, human HEV-7 infections might contribute to the HEV prevalence in all studied areas, where camel products are frequent parts of human diet (15). A foodborne transmission scenario is further suggested by the fact that 1 of 12 positive serum in the study was actually sampled in a slaughterhouse, documenting that meat from infected animals can enter the food chain (6). Detections of HEV-7 RNA in feces in this and a previous study (2) point at feces or feces-contaminated camel products, such as milk, as putative additional sources of human infection. Considering the importance of dromedaries as livestock animals (15), risk groups, such as slaughterhouse workers, should be screened for HEV-7 infection. 


\section{Acknowledgments}

We thank Monika Eschbach-Bludau, Sebastian Brünink, and Tobias Bleicker for providing excellent technical assistance.

This study was supported by the European Commission (project COMPARE), the German Research Foundation (project DR772/12-1). A.L. and V.C.M were supported by the Centrum of International Migration and Development (Contract No. 81195004). The funders had no role in study design, data collection and analysis, decision to publish, or preparation of the manuscript.

Mrs. Rasche is a doctoral student at the Institute of Virology, Bonn, Germany. Her primary research interests include detection and characterization of novel zoonotic hepatitis viruses.

\section{References}

1. Aggarwal R. The global prevalence of hepatitis $E$ virus infection and susceptibility: a systematic review [cited 2016 May 16]. http://whqlibdoc.who.int/hq/2010/WHO_IVB_10.14_eng.pdf

2. Woo PC, Lau SK, Teng JL, Tsang AK, Joseph M, Wong EY, et al. New hepatitis E virus genotype in camels, the Middle East. Emerg Infect Dis. 2014;20:1044-8. http://dx.doi.org/10.3201/ eid2006.140140

3. Smith DB, Simmonds P, Jameel S, Emerson SU, Harrison TJ, Meng XJ, et al. Consensus proposals for classification of the family Hepeviridae. J Gen Virol. 2014;95:2223-32. http://dx.doi.org/ 10.1099/vir.0.068429-0

4. Lee GH, Tan BH, Chi-Yuan Teo E, Lim SG, Dan YY, Wee A, et al. Chronic infection with camelid hepatitis $\mathrm{E}$ virus in a liver transplant recipient who regularly consumes camel meat and milk. Gastroenterology. 2016;150:355-357.e3. http://dx.doi.org/ $10.1053 /$ j.gastro.2015.10.048

5. Corman VM, Jores J, Meyer B, Younan M, Liljander A, Said MY, et al. Antibodies against MERS coronavirus in dromedary camels, Kenya, 1992-2013. Emerg Infect Dis. 2014;20:1319-22. http://dx.doi.org/10.3201/eid2008.140596

6. Müller MA, Corman VM, Jores J, Meyer B, Younan M, Liljander A, et al. MERS coronavirus neutralizing antibodies in camels, Eastern Africa, 1983-1997. Emerg Infect Dis. 2014; 20:2093-5. http://dx.doi.org/10.3201/eid2012.141026
7. Meyer B, Müller MA, Corman VM, Reusken CB, Ritz D, Godeke GJ, et al. Antibodies against MERS coronavirus in dromedary camels, United Arab Emirates, 2003 and 2013. Emerg Infect Dis. 2014;20:552-9. http://dx.doi.org/10.3201/ eid2004.131746

8. Drexler JF, Seelen A, Corman VM, Fumie Tateno A, Cottontail V, Melim Zerbinati R, et al. Bats worldwide carry hepatitis E virus-related viruses that form a putative novel genus within the family Hepeviridae. J Virol. 2012;86:9134-47. http://dx.doi.org/ 10.1128/JVI.00800-12

9. Girón-Callejas A, Clark G, Irving WL, McClure CP. In silico and in vitro interrogation of a widely used HEV RT-qPCR assay for detection of the species orthohepevirus A. J Virol Methods. 2015;214:25-8. http://dx.doi.org/10.1016/j.jviromet.2014.11.025

10. Baylis SA, Blümel J, Mizusawa S, Matsubayashi K, Sakata H, Okada Y, et al.. World Health Organization International Standard to harmonize assays for detection of hepatitis $\mathrm{E}$ virus RNA. Emerg Infect Dis. 2013;19:729-35. http://dx.doi.org/ 10.3201/eid1905.121845

11. Smith DB, Simmonds P; International Committee on Taxonomy of Viruses Hepeviridae Study Group, Jameel S, Emerson SU, Harrison TJ, et al. Consensus proposals for classification of the family Hepeviridae. J Gen Virol. 2014;95(Pt 10):2223-32. http://dx.doi.org/10.1099/vir.0.068429-0

12. Zhou X, Kataoka M, Liu Z, Takeda N, Wakita T, Li TC. Characterization of self-assembled virus-like particles of dromedary camel hepatitis E virus generated by recombinant baculoviruses. Virus Res. 2015;210:8-17. http://dx.doi.org/ 10.1016/j.virusres.2015.06.022

13. Krumbholz A, Joel S, Neubert A, Dremsek P, Dürrwald R, Johne R, et al. Age-related and regional differences in the prevalence of hepatitis E virus-specific antibodies in pigs in Germany. Vet Microbiol. 2013;167:394-402. http://dx.doi.org/10.1016/ j.vetmic.2013.10.001

14. Smith DB, Simmonds P, Izopet J, Oliveira-Filho EF, Ulrich RG, Johne R, et al. Proposed reference sequences for hepatitis E virus subtypes. J Gen Virol. 2016;97:537-42. http://dx.doi.org/10.1099/ jgv.0.000393

15. Kadim IT, Mahgoub O, Faye B, Farouk MM, editors. Camel meat and meat products Wallingford (UK): CAB International; 2013.

Address for correspondence: Christian Drosten, Institute of Virology, University of Bonn Medical Centre, Sigmund Freud Str 25, 53105 Bonn, Germany; email: drosten@virology-bonn.de

\section{EID Podcast: Rat Lungworm Expands into North America}

The rat lungworm, Angiostrongylus (Parastrongylus) cantonensis, causes eosinophilic meningitis in humans and various disease manifestations in atypical host species, including wildlife and captive animals.

\section{Emily York, integrated pest management specialist at the Sam Noble Museum of Natural History, discusses the rat lungworm expansion in North America.}

Cotton rat, Sigmodon hispidus. Photo courtesy Public Health Image Library.

\section{Visit our website to listen: http://www2c.cdc.gov/podcasts/player.asp?f=8640172}

